\title{
Type Synthesis of 1T2R Parallel Mechanisms Using Structure Coupling-Reducing Method
}

\author{
Haitao Liu ${ }^{1 *} \mathbb{D}, K_{e} X^{1}$, Huiping Shen ${ }^{2}$, Xianlei Shan ${ }^{1}$ and Tingli Yang ${ }^{2}$
}

\begin{abstract}
Direct kinematics with analytic solutions is critical to the real-time control of parallel mechanisms. Therefore, the type synthesis of a mechanism having explicit form of forward kinematics has become a topic of interest. Based on this purpose, this paper deals with the type synthesis of 1T2R parallel mechanisms by investigating the topological structure coupling-reducing of the 3UPS\&UP parallel mechanism. With the aid of the theory of mechanism topology, the analysis of the topological characteristics of the 3UPS\&UP parallel mechanism is presented, which shows that there are highly coupled motions and constraints amongst the limbs of the mechanism. Three methods for structure coupling-reducing of the 3UPS\&UP parallel mechanism are proposed, resulting in eight new types of 1T2R parallel mechanisms with one or zero coupling degree. One obtained parallel mechanism is taken as an example to demonstrate that a mechanism with zero coupling degree has an explicit form for forward kinematics. The process of type synthesis is in the order of permutation and combination; therefore, there are no omissions. This method is also applicable to other configurations, and novel topological structures having simple forward kinematics can be obtained from an original mechanism via this method.
\end{abstract}

Keywords: Type synthesis, Structure coupling-reducing, Coupling degree, Parallel mechanism

\section{Introduction}

Forward kinematics, which is one of the fundamental issues in the kinematic analysis of a parallel mechanism (PM) [1], refers to evaluating the pose of a platform from a set of specified values of actuated joint parameters. Generally, the forward kinematic analysis of a PM is much more complex than its reverse process, i.e., inverse kinematics. The difficulty is mainly in relation to the dependent motions of the passive joints in the mechanism.

For a better illustration, three planar open kinematic chains that can be employed to compose planar PMs are given as examples (see Figure 1). The first one is a 2-degree-of-freedom (DOF) planar open kinematic chain applying a constraint on a moving platform. It is easy to see that given the position of point $A$, the lengths of the linkages, and input angle $\theta$, the position of point $D$ on

\footnotetext{
*Correspondence: liuht@tju.edu.cn

${ }^{1}$ Key Laboratory of Mechanism Theory and Equipment Design, Ministry of Education, Tianjin University, Tianjin 300350, China

Full list of author information is available at the end of the article
}

the moving platform can be directly determined using the vector pointing from point $A$ to point $D$. Meanwhile, for the second and third cases, because of the need to evaluate passive joint parameters $\alpha^{*}$ and $\beta^{*}$, the forward kinematics of point $D$ cannot be solved using only the kinematic equations formulated by the open kinematic chain itself, and additional equations depicting the coupled motions among the chains of the PM have to be derived. Therefore, the higher the number of passive joints in a PM, the higher the complexity of the forward kinematic analysis. In order to calculate the forward kinematic problem of a PM efficiently, great efforts have been made to develop numerical algorithms for particular case studies [2-8]. Although the real-time performance benefits of these methods have been exemplified, only approximate solutions for forward kinematics have so far been attained.

Because this issue is critical to the real-time control of PMs [9], the type synthesis of a mechanism having an explicit form for forward kinematics has become a topic of interest. However, the majority of currently known type syntheses aim to realize output motion only [10-12]. 


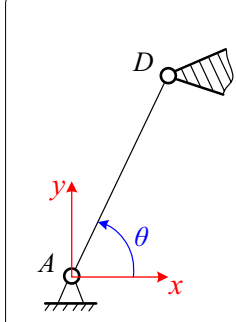

a

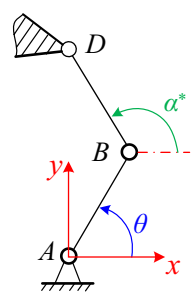

b

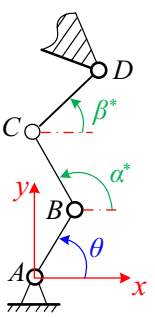

c
Figure 1 Open kinematic chains

To design PMs with analytic direct kinematics, a metric for assessing the complexity of forward kinematics at a topological level has to first be defined. For this purpose, the motion of coupling degree, which is denoted by $\kappa$, is proposed in the theory of mechanism topology [13, 14], giving the minimum number of passive joint parameters that need to be solved in the process of forward kinematic analysis. For a specific PM, the value of $\kappa$ is usually an integral number larger than zero. However, when its value is equal to zero, analytical solutions for forward kinematics can be achieved. Through further examination of the coupling degree of a PM, the approach known as structure coupling-reducing (SCR), which aims to reduce the coupling degree while keeping the DOF and position and orientation characteristics (POC) [14] of the mechanism unchanged, is developed in $[15,16]$. In this manner, new topological structures that have simple forward kinematics can be obtained from an original mechanism by rearranging the axes and positions of the joints. With the aid of this approach, eight new 3-DOF translational PMs [17], three types of 3T1R PMs [18], and sixteen novel 6-DOF PMs [19-23] have been synthesized.

Drawing on the SCR method, this paper deals with the type synthesis of 1T2R PMs by reducing the coupling degree of the 3UPS\&UP PM within the Tricept robot [24-26]. The rest of this paper is organized as follows. In Section 2, the topological characteristics of the 3UPS\&UP PM are systemically analyzed, followed by the calculation of the coupling degree of the mechanism. In Section 3, the SCR methods are then introduced to synthesize 1T2R PMs with new topologies. Finally, in Section 4, the forward kinematic analysis of one of the obtained PMs is performed to demonstrate the validity of the method, before conclusions are drawn in Section 5 .

\section{Topological Characteristics Analysis}

In this section, the topological characteristics [13, 14] in terms of the POC set, decomposition of single-open chains (SOC), constraint degree $\Delta$, and coupling degree $\kappa$ of the 3UPS\&UP PM within the Tricept robot are systematically analyzed.

\subsection{Mobility Analysis}

As shown in Figure 2, the 3UPS\&UP PM consists of three active limbs and one passive limb connecting the base to the moving platform. The active limb $i(i=1,2,3)$ is composed of a universal joint $\mathrm{U}_{i}$, a prismatic joint $\mathrm{P}_{i}$, and a spherical joint $S_{i}$. Meanwhile, the passive limb is composed of a universal joint $\mathrm{U}_{4}$ and a prismatic joint $\mathrm{P}_{4}$.

Based on the theory of mechanism topology [14], the POC sets indicating the motion characteristics of the $U$, P, and S joints (see Figure 3) can be expressed as follows:

$$
\begin{aligned}
& M_{\mathrm{U} i}=\left[\begin{array}{l}
\left\{t^{2}\left(\perp \boldsymbol{\rho}_{\mathrm{U} i}\right)\right\} \\
r^{2}\left(\| \mathrm{U}_{i}\right)
\end{array}\right], M_{\mathrm{P} i}=\left[\begin{array}{l}
t^{1}\left(\| \mathrm{P}_{i}\right) \\
r^{0}
\end{array}\right], \\
& M_{\mathrm{S} i}=\left[\begin{array}{l}
\left\{t^{2}\left(\perp \boldsymbol{\rho}_{\mathrm{S} i}\right)\right\} \\
r^{3}
\end{array}\right],
\end{aligned}
$$

where $t$ and $r$ represent translation and rotation, respectively; $\boldsymbol{\rho}_{\mathrm{U} i}$ and $\boldsymbol{\rho}_{\mathrm{S} i}$ are the vectors from the centers of $\mathrm{U}_{i}$ and $S_{i}$ to a reference point $O$, respectively; the superscript is the number of translational/rotational motion; $(*)$

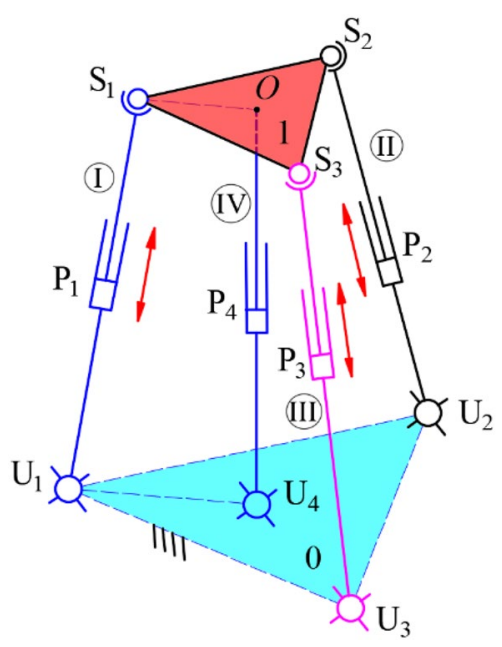

Figure 2 Schematic diagram of 3UPS\&UP PM

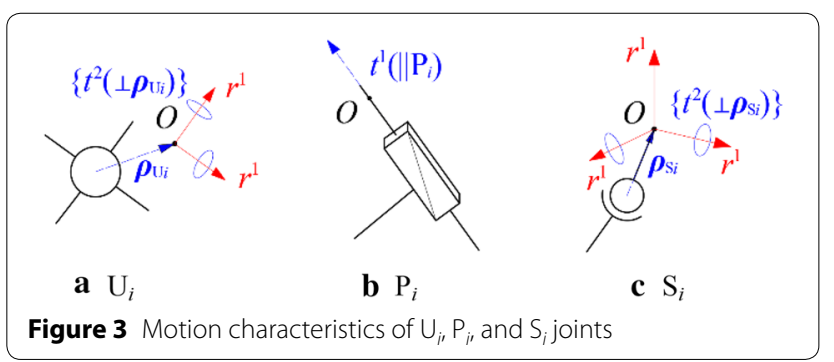


describes the direction of translation or the axis of rotation; while $\left\{{ }^{*}\right\}$ denotes the parasitic translations induced by rotations. For example, $t^{1}\left(\| \mathrm{P}_{i}\right)$ and $r^{0}$ in $M_{\mathrm{P} i}$ indicate that a prismatic joint $\mathrm{P}_{i}$ can achieve only one translational motion parallel to the direction of the joint.

The joints are serially connected in a limb, and therefore, the POC set of a limb can be expressed as the union of the POC sets of all joints. Here, the operation rules of "union" are presented in Refs. [27, 28]. The POC sets of UPS and UP limbs can then be obtained via

$$
\begin{aligned}
M_{b i} & =M_{\mathrm{U} i} \cup M_{\mathrm{P} i} \cup M_{\mathrm{S} i} \\
& =\left[\begin{array}{l}
\left\{t^{2}\left(\perp \boldsymbol{\rho}_{\mathrm{U} i}\right)\right\} \\
r^{2}\left(\| \mathrm{U}_{i}\right)
\end{array}\right] \cup\left[\begin{array}{l}
t^{1}\left(\| \mathrm{P}_{i}\right) \\
r^{0}
\end{array}\right] \cup\left[\begin{array}{l}
\left\{t^{2}\left(\perp \boldsymbol{\rho}_{\mathrm{S} i}\right)\right\} \\
r^{3}
\end{array}\right] \\
& =\left[\begin{array}{l}
t^{1}\left(\| \mathrm{P}_{i}\right) \\
r^{0}
\end{array}\right] \cup\left[\begin{array}{l}
\left\{t^{2}\left(\perp \boldsymbol{\rho}_{\mathrm{U} i}\right)\right\} \cup\left\{t^{2}\left(\perp \boldsymbol{\rho}_{\mathrm{S} i}\right)\right\} \\
r^{2}\left(\| \mathrm{U}_{i}\right) \cup r^{3}
\end{array}\right] \\
& =\left[\begin{array}{l}
t^{1}\left(\| \mathrm{P}_{i}\right) \cup t^{2}\left(\perp \boldsymbol{\rho}_{\mathrm{U} i}\right) \\
r^{3}
\end{array}\right]=\left[\begin{array}{l}
t^{3} \\
r^{3}
\end{array}\right], \quad i=1,2,3, \\
M_{b 4} & =M_{\mathrm{U} 4} \cup M_{\mathrm{P} 4}=\left[\begin{array}{l}
\left\{t^{2}\left(\perp \boldsymbol{\rho}_{\mathrm{U} 4}\right)\right\} \\
r^{2}\left(\| \mathrm{U}_{4}\right)
\end{array}\right] \cup\left[\begin{array}{l}
t^{1}\left(\| \mathrm{P}_{4}\right) \\
r^{0}
\end{array}\right] \\
& =\left[\begin{array}{l}
t^{1}\left(\| \mathrm{P}_{4}\right) \cup\left\{t^{2}\left(\perp \boldsymbol{\rho}_{\mathrm{U} 4}\right)\right\} \\
r^{2}\left(\| \mathrm{U}_{4}\right)
\end{array}\right] .
\end{aligned}
$$

In Eq. (2), because the rotational axes of $S_{i}$ could be parallel to those of $\mathrm{U}_{i}$, the union of $\left\{t^{2}\left(\perp \boldsymbol{\rho}_{\mathrm{U} i}\right)\right\}$ and $\left\{t^{2}\left(\perp \boldsymbol{\rho}_{\mathrm{S} i}\right)\right\}$ leads to $t^{2}\left(\perp \boldsymbol{\rho}_{\mathrm{U} i}\right)$, according to the rules given in Ref. [21]. Furthermore, because the directions of $t^{2}\left(\perp \boldsymbol{\rho}_{\mathrm{U} i}\right)$ are perpendicular to the direction of $\mathrm{P}_{i}$, the union of $t^{2}\left(\perp \boldsymbol{\rho}_{i}\right)$ and $t^{1}\left(\| \mathrm{P}_{i}\right)$ results in $t^{3}$. In Eq. (3), the translational directions of $\left\{t^{2}\left(\perp \boldsymbol{\rho}_{\mathrm{U} 4}\right)\right\}$ and $t^{1}\left(\| \mathrm{P}_{4}\right)$ are independent; therefore, the union of these two translational motions is $t^{1}\left(|| \mathrm{P}_{4}\right) \cup\left\{t^{2}\left(\perp \rho_{\mathrm{U} 4}\right)\right\}$. From Eqs. (2) and (3), it can be found that the UPS limb is a 6-DOF unconstrained open kinematic chains, while the UP limb has one translational and two rotational independent motions.

Because of the parallel topology of the mechanism, the POC set of the moving platform, $M_{p a}$, can be obtained by taking the intersection of the POC sets of the limbs. Based on the operation rules of "intersection" given in Refs. [27, 28], the POC set is defined as follows:

$$
\begin{aligned}
M_{p a} & =\bigcap_{i=1}^{4} M_{b i} \\
& =\left[\begin{array}{l}
t^{1}\left(\| \mathrm{P}_{4}\right) \cup\left\{t^{2}\left(\perp \mathrm{U}_{4}\right)\right\} \\
r^{2}\left(\| \mathrm{U}_{4}\right)
\end{array}\right] \cap\left[\begin{array}{c}
t^{3} \\
r^{3}
\end{array}\right] \cap\left[\begin{array}{c}
t^{3} \\
r^{3}
\end{array}\right] \cap\left[\begin{array}{l}
t^{3} \\
r^{3}
\end{array}\right] \\
& =\left[\begin{array}{l}
t^{1}\left(\| \mathrm{P}_{4}\right) \cup\left\{t^{2}\left(\perp \mathrm{U}_{4}\right)\right\} \\
r^{2}\left(\| \mathrm{U}_{4}\right)
\end{array}\right],
\end{aligned}
$$

from which it can be seen that $M_{p a}$ is identical with $M_{b 4}$. For this reason, the UP limb is also known as the POC chain [29].

\subsection{Coupling Degree}

The coupling degree can be considered as a metric for assessing the complexity of forward kinematics. For the evaluation of coupling degree of the PM, the mechanism must be decomposed into several SOCs and independent loops [28]. As shown in Figure 4, the decomposition is given as follows:

$$
\begin{aligned}
& \mathrm{SOC}_{1}\left\{\mathrm{U}_{1}-\mathrm{P}_{1}-\mathrm{S}_{1}-\mathrm{P}_{4}-\mathrm{U}_{4}\right\} \\
& \mathrm{SOC}_{2}\left\{\mathrm{U}_{2}-\mathrm{P}_{2}-\mathrm{S}_{2}\right\}, \mathrm{SOC}_{3}\left\{\mathrm{U}_{3}-\mathrm{P}_{3}-\mathrm{S}_{3}\right\}
\end{aligned}
$$

The first independent loop, Loop1, can then be defined by fixing both end links of $\mathrm{SOC}_{1}$ according to Ref. [14], while the second independent loop, Loop2, is obtained by taking Loop1 as a sub-mechanism and attaching two end links of $\mathrm{SOC}_{2}$ to Loop1. Analogously, the third independent loop, Loop3, is defined by attaching two end links of $\mathrm{SOC}_{3}$ to Loop2 (see Figure 4). With these definitions, the

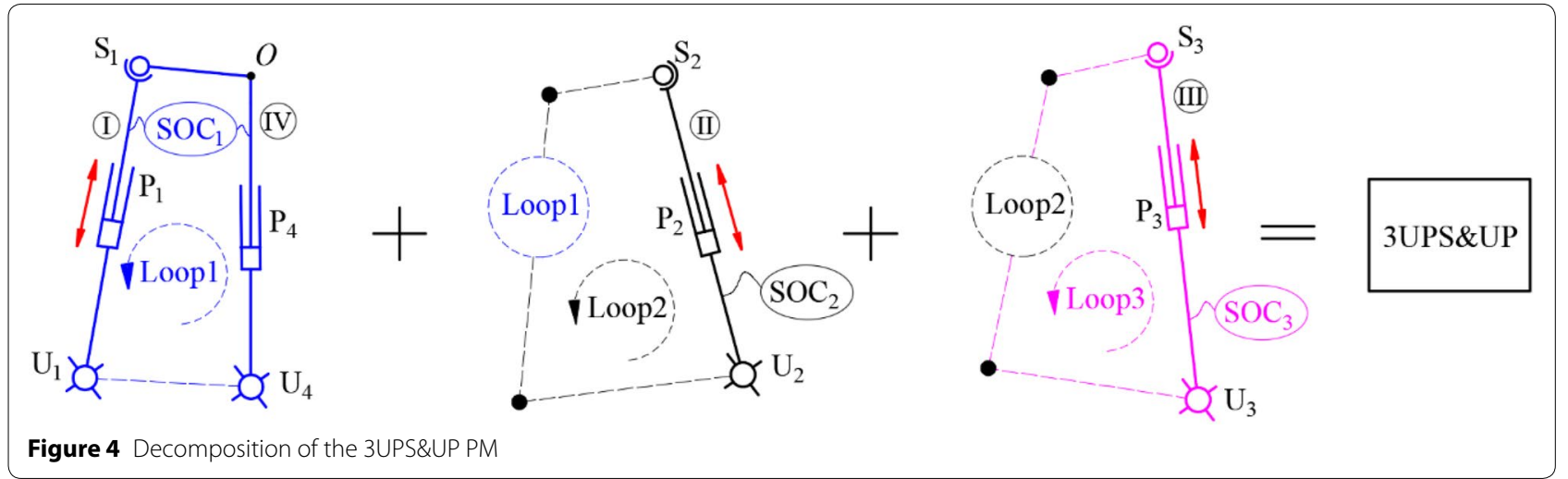


number of independent displacement equations of the three loops can be computed following the method presented in Ref. [28].

$$
\begin{aligned}
\xi_{L 1} & =\operatorname{dim}\left\{M_{b_{1}} \cup M_{b 4}\right\} \\
& =\operatorname{dim}\left\{\left[\begin{array}{l}
t^{1}\left(\| \mathrm{P}_{4}\right) \cup\left\{t^{2}\left(\perp \mathrm{U}_{4}\right)\right\} \\
r^{2}\left(\| \mathrm{U}_{4}\right)
\end{array}\right] \cup\left[\begin{array}{l}
t^{3} \\
r^{3}
\end{array}\right]\right\} \quad(5) \\
& =\operatorname{dim}\left\{\left[\begin{array}{l}
t^{3} \\
r^{3}
\end{array}\right]\right\}=3+3=6, \\
\xi_{L 2} & =\operatorname{dim}\left\{\left(M_{b_{1}} \cap M_{b 4}\right) \cup M_{b 2}\right\} \\
& =\operatorname{dim}\left\{\left(\left[\begin{array}{c}
t^{1}\left(\| \mathrm{P}_{4}\right) \cup\left\{t^{2}\left(\perp \mathrm{U}_{4}\right)\right\} \\
r^{2}\left(\| \mathrm{U}_{4}\right)
\end{array}\right] \cap\left[\begin{array}{c}
t^{3} \\
r^{3}
\end{array}\right]\right) \cup\left[\begin{array}{l}
t^{3} \\
r^{3}
\end{array}\right]\right\} \\
& =\operatorname{dim}\left\{\left[\begin{array}{c}
t^{3} \\
r^{3}
\end{array}\right]\right\}=3+3=6,
\end{aligned}
$$$$
\xi_{L 3}=\operatorname{dim}\left\{\left(M_{b_{1}} \cap M_{b 4} \cap M_{b 2}\right) \cup M_{b 3}\right\}
$$$$
=\operatorname{dim}\left\{\left(\left[\begin{array}{l}
t^{1}\left(|| \mathrm{P}_{4}\right) \cup\left\{t^{2}\left(\perp \mathrm{U}_{4}\right)\right\} \\
r^{2}\left(\| \mathrm{U}_{4}\right)
\end{array}\right]\right.\right.
$$$$
\left.\left.\cap\left[\begin{array}{l}
t^{3} \\
r^{3}
\end{array}\right] \cap\left[\begin{array}{l}
t^{3} \\
r^{3}
\end{array}\right]\right) \cup\left[\begin{array}{l}
t^{3} \\
r^{3}
\end{array}\right]\right\}
$$$$
=\operatorname{dim}\left\{\left[\begin{array}{l}
t^{3} \\
r^{3}
\end{array}\right]\right\}=3+3=6 \text {, }
$$

where $\operatorname{dim}\{*\}$ denotes the dimension, i.e., the number of independent motions, of a POC set. Subsequently, to evaluate the coupling degree, the constraint degree describing the constraint relationship of a SOC has to be calculated [14]. For this specific case, the constraint degrees of the three SOCs are

$$
\begin{aligned}
& \Delta_{1}=\sum_{i=1}^{5} f_{i}-I_{1}-\xi_{L_{1}}=9-1-6=2, \\
& \Delta_{2}=\sum_{i=1}^{3} f_{i}-I_{2}-\xi_{L_{2}}=6-1-6=-1, \\
& \Delta_{3}=\sum_{i=1}^{3} f_{i}-I_{3}-\xi_{L_{3}}=6-1-6=-1,
\end{aligned}
$$

where $f_{i}$ is the DOF of the $i$ th joint, and $I_{j}$ is the number of actuated joints in the $j$ th $\operatorname{SOC}(j=1,2,3)$. The constraint degree of $\mathrm{SOC}_{1}$ is shown to be 2 , which means that two passive joint parameters have to be solved to determine the motion of $\mathrm{SOC}_{1}$. Meanwhile, the constraint degree of $\mathrm{SOC}_{2}\left(\mathrm{SOC}_{3}\right)$ is -1 , indicating that a constraint equation should be established to solve one of the passive joint parameters in the kinematic equations of $\mathrm{SOC}_{1}$. Finally, the coupling degree of the 3UPS\&UP PM can be evaluated via [14]

$$
\kappa=\frac{1}{2} \sum_{j=1}^{3}\left|\Delta_{j}\right|=\frac{1}{2}(2+|-1|+|-1|)=2 .
$$

This relationship demonstrates that the constraints of the three SOCs are highly coupled. Therefore, there is no analytic solution for the forward kinematics of the 3UPS\&UP PM. Approaches for the type synthesis of new topological structures with the same topological characteristics but using simple forward kinematics are investigated in the following section.

\section{Type Synthesis}

Three SCR methods, which aim to reduce the coupling degree of the 3UPS\&UP PM and obtain new mechanisms that have simple forward kinematics, are presented in this section.

\subsection{Method One: Changing the Passive POC Chain into Active Chain}

In the 3UPS\&UP PM, the UP limb is the POC chain because of $M_{b 4}$ being identical with $M_{p a}$. When the passive prismatic joint in the POC chain is changed into an active one (see Figure 5), the constraint degree $\Delta_{1}$ of $\mathrm{SOC}_{1}$ can be reduced to

$$
\begin{aligned}
& \mathrm{SOC}_{1}\left\{\mathrm{U}_{1}-\mathrm{P}_{1}-\mathrm{S}_{1}-\mathrm{P}_{4}-\mathrm{U}_{4}\right\}, \\
& \Delta_{1}=\sum_{i=1}^{5} f_{i}-I_{1}-\xi_{L_{1}}=9-2-6=1,
\end{aligned}
$$

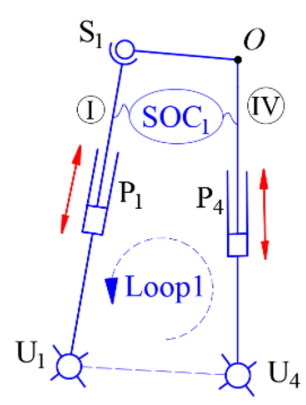

Figure 5 The first loop 
because $I_{1}=2$ in this equation. Furthermore, in order to achieve a $\mathrm{PM}$ without redundant actuation, the active joint $\mathrm{P}_{3}$ in $\mathrm{SOC}_{3}$ is taken as a passive joint, resulting in

$$
\begin{aligned}
& \mathrm{SOC}_{3}\left\{\mathrm{U}_{3}-\mathrm{P}_{3}-\mathrm{S}_{3}\right\}, \\
& \Delta_{3}=\sum_{i=1}^{3} f_{i}-I_{3}-\xi_{L_{3}}=6-0-6=0 .
\end{aligned}
$$

Hence, the coupling degree of this new PM (No.1 (a) in Table 1) is

$$
\kappa=\frac{1}{2} \sum_{j=1}^{3}\left|\Delta_{j}\right|=\frac{1}{2}(1+|-1|+|0|)=1,
$$

indicating that only one passive joint parameter needs to be solved in the forward kinematics. Because limb 3 becomes a 6-DOF passive limb in this special case, it has no effect on $M_{p a}$. Therefore, it is removable, leading to the PM (No.1 $(b)$ in Table 1) within the TriVariant robot [30].

\subsection{Method Two: Making the Centers of Spherical Joints Coincident}

Another approach to reduce the coupling degree is by making the centers of the spherical joints on the moving platform coincident. For example, the centers of spherical joints $S_{1}$ and $S_{3}$ are coincident, as shown in No. 3 in Table 1. The SOCs of the obtained mechanism (see Figure 6) and their constraint degrees can then be redefined as

$$
\begin{aligned}
& \mathrm{SOC}_{1}\left\{\mathrm{U}_{1}-\mathrm{P}_{1}-\mathrm{S}_{13}-\mathrm{P}_{3}-\mathrm{U}_{3}\right\}, \\
& \Delta_{1}=\sum_{i=1}^{5} f_{i}-I_{1}-\xi_{L_{1}}=9-2-6=1, \\
& \mathrm{SOC}_{2}\left\{\mathrm{U}_{4}-\mathrm{P}_{4}\right\} \\
& \Delta_{2}=\sum_{i=1}^{2} f_{i}-I_{2}-\xi_{L_{2}}=3-3=0, \\
& \mathrm{SOC}_{3}\left\{\mathrm{U}_{2}-\mathrm{P}_{2}-\mathrm{S}_{2}\right\}, \\
& \Delta_{3}=\sum_{i=1}^{3} f_{i}-I_{3}-\xi_{L_{3}}=6-1-6=-1,
\end{aligned}
$$

resulting in the coupling degree of this new mechanism:

$$
\kappa=\frac{1}{2} \sum_{j=1}^{3}\left|\Delta_{j}\right|=\frac{1}{2}(|1|+|0|+|-1|)=1 .
$$

Therefore, a new type of 1T2R PM with lower coupling degree is synthesized. In addition, we can also make the centers of three spherical joints connected to the moving platform coincident. In this way, a new mechanism, as shown in No.4 in Table 1, can then be obtained. For this mechanism, the redefined SOCs and their constraint degrees are given as follows:

$$
\begin{aligned}
& \mathrm{SOC}_{1}\left\{\mathrm{U}_{1}-\mathrm{P}_{1}-\mathrm{S}_{123}-\mathrm{P}_{3}-\mathrm{U}_{3}\right\}, \\
& \Delta_{1}=\sum_{i=1}^{5} f_{i}-I_{1}-\xi_{L_{1}}=8-2-6=0, \\
& \mathrm{SOC}_{2}\left\{\mathrm{U}_{4}-\mathrm{P}_{4}\right\}, \Delta_{2}=\sum_{i=1}^{2} f_{i}-I_{2}-\xi_{L_{2}}=3-3=0, \\
& \mathrm{SOC}_{3}\left\{\mathrm{U}_{2}-\mathrm{P}_{2}-\mathrm{R}^{(\mathrm{U} 1-\mathrm{U} 3)}\right\}, \\
& \quad \Delta_{3}=\sum_{i=1}^{3} f_{i}-I_{3}-\xi_{L_{3}}=4-1-3=0 .
\end{aligned}
$$

Here, the spherical joint $\mathrm{S}_{2}$ in $\mathrm{SOC}_{3}$ is degenerated into a revolute joint $\mathrm{R}^{(\mathrm{U} 1-\mathrm{U} 3)}$ with the rotational axis parallel to the line passing through the centers of joints $U_{1}$ and $U_{3}$. The coupling degree of this mechanism is then

$$
\kappa=\frac{1}{2} \sum_{j=1}^{3}\left|\Delta_{j}\right|=\frac{1}{2}(|0|+|0|+|0|)=0 .
$$

Because $\kappa=0$, it can be concluded that the PM has an explicit form for forward kinematics.

\subsection{Method Three: Integrating the Rotational Axes of Universal Joints}

This method aims to directly reduce $\sum f_{i}$ in a SOC. Note that the joint $U$ can be decomposed into a proximal revolute joint $\mathrm{R}^{\prime}$ and a distal revolute joint $\mathrm{R}$ with their rotational axes being perpendicular. Hence, in the 3UPS\&UP $P M$, the revolute joints $R_{2}^{\prime}$ and $R_{4}^{\prime}$ of $U_{2}$ and $U_{4}$, respectively, could be made coincident and integrated into a revolute joint $R_{24}$ (see No. 5 in Table 1). The kinematic chains $\mathrm{U}_{4} \mathrm{P}_{4}$ and $\mathrm{U}_{2} \mathrm{P}_{2} \mathrm{~S}_{2}$ can then be regarded as a hybrid singleopen chain, $\mathrm{HSOC}_{1}[15]$.

$$
\mathrm{HSOC}_{1}\left\{\mathrm{R}_{24}-\left(\mathrm{R}_{2} \perp \mathrm{P}_{2}-\mathrm{S}_{2}\right)\left(\mathrm{R}_{4} \perp \mathrm{P}_{4}\right)\right\} \text {. }
$$

The SOCs and corresponding constraint degrees of this mechanism (see Figure 7) can then be defined as

$$
\begin{aligned}
& \mathrm{SOC}_{1}\left\{\mathrm{HSOC}_{1}-\mathrm{S}_{3}-\mathrm{P}_{3}-\mathrm{U}_{3}\right\}, \\
& \Delta_{1}=\sum_{i=1}^{5} f_{i}-I_{1}-\xi_{L_{1}}=9-2-6=1,
\end{aligned}
$$


Table 1 Topological structure obtained via design of SCR for 3UPS\&UP

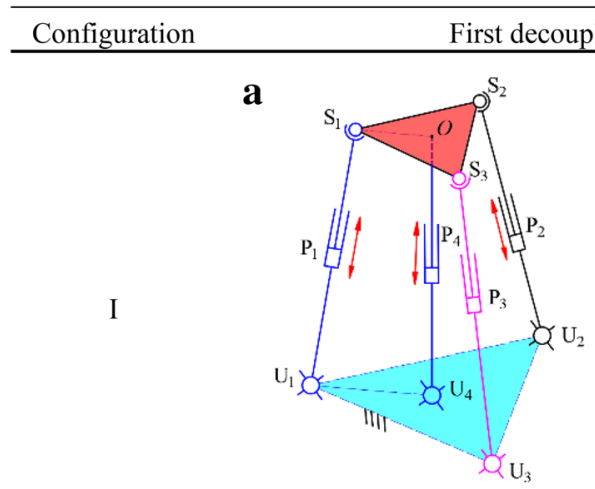

No. 1 PM $[F=3, v=2, \kappa=1]$

II

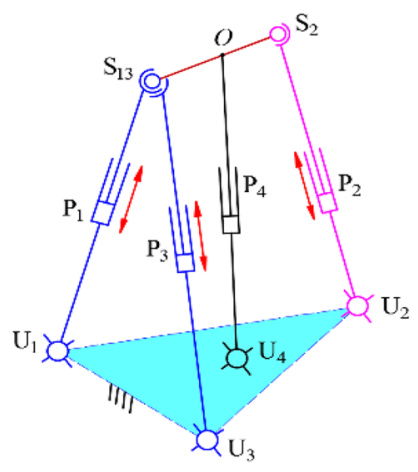

No. 3 PM $[F=3, v=3, \kappa=1]$

III

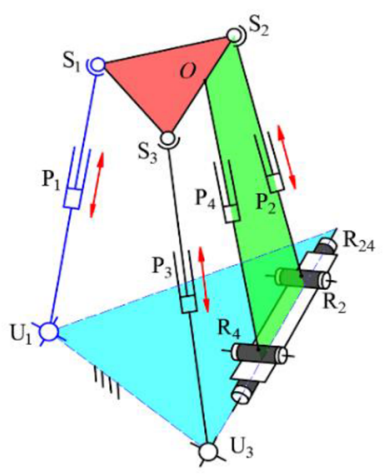

No. 5 PM $[F=3, v=2, \kappa=1]$
Second decoupling $(\kappa=1 \rightarrow \kappa=0)$

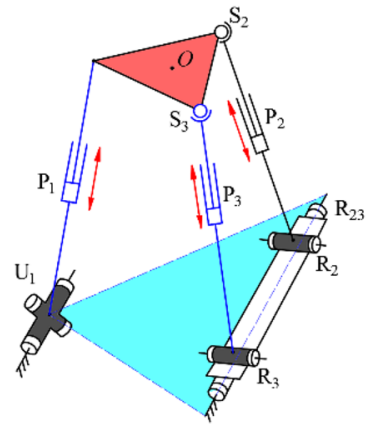

No. $2 \operatorname{PM}[F=3, v=1, \kappa=0]$

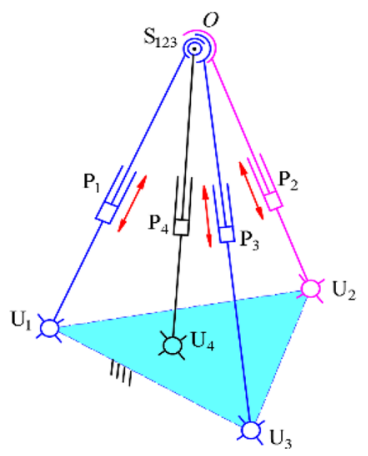

No. $4 \mathrm{PM}[F=3, v=3, \kappa=0]$

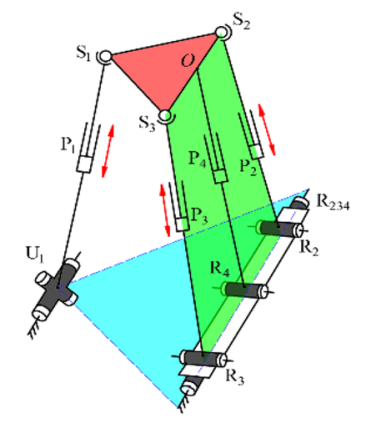

No. $6 \mathrm{PM}[F=3, v=1, \kappa=0]$

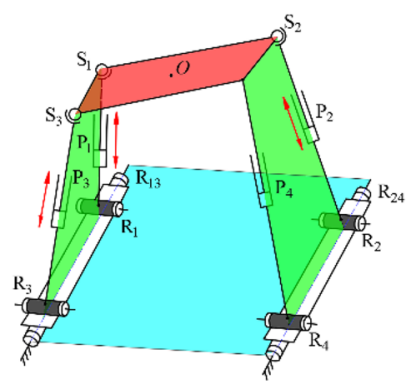

No. 7 PM $[F=3, v=1, \kappa=0]$ 


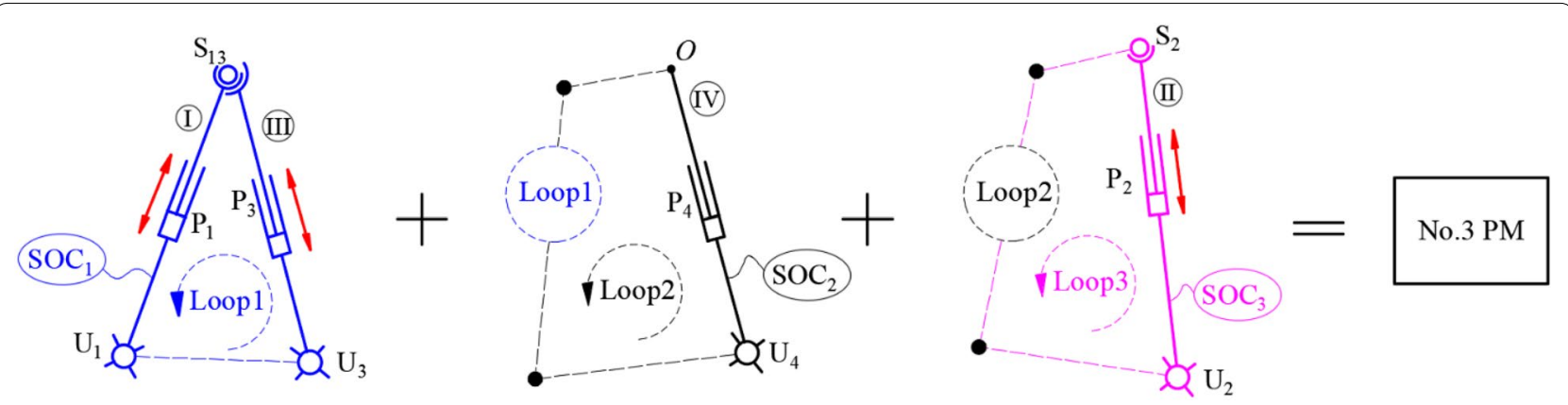

Figure 6 Topological decomposition of No. 3 PM

$$
\begin{aligned}
& \mathrm{SOC}_{2}\left\{\mathrm{U}_{1}-\mathrm{P}_{1}-\mathrm{S}_{1}\right\}, \\
& \Delta_{2}=\sum_{i=1}^{2} f_{i}-I_{2}-\xi_{L_{2}}=6-1-6=-1 .
\end{aligned}
$$

Consequently, the coupling degree of the new PM is

$$
\kappa=\frac{1}{2} \sum_{j=1}^{2}\left|\Delta_{j}\right|=\frac{1}{2}(|1|+|-1|)=1 .
$$

Furthermore, $\mathrm{R}_{2}^{\prime}, \mathrm{R}_{3}^{\prime}$, and $\mathrm{R}_{4}^{\prime}$ of $\mathrm{U}_{2}, \mathrm{U}_{3}$, and $\mathrm{U}_{4}$, respectively, can also be integrated into one revolute joint $R_{234}$. In this manner, the kinematic chains $\mathrm{U}_{4} \mathrm{P}_{4}, \mathrm{U}_{3} \mathrm{P}_{3} \mathrm{~S}_{3}$, and $\mathrm{U}_{2} \mathrm{P}_{2} \mathrm{~S}_{2}$ are converted into another $\mathrm{HSOC}_{1}$,

$\mathrm{HSOC}_{1}\left\{\mathrm{R}_{234}-\left(\mathrm{R}_{4} \perp \mathrm{P}_{4}\right)\left(\mathrm{R}_{2} \perp \mathrm{P}_{2}-\mathrm{S}_{2}\right)\left(\mathrm{R}_{3} \perp \mathrm{P}_{3}-\mathrm{S}_{3}\right)\right\}$, resulting in the R(2RPS\&RP)\&UPS PM (No.6 in Table 1 ) within the TriMule robot [31]. For this mechanism, its SOC can be expressed as

$$
\begin{aligned}
& \mathrm{SOC}_{1}\left\{\mathrm{HSOC}_{1}-\mathrm{S}_{1}-\mathrm{P}_{1}-\mathrm{U}_{1}\right\}, \\
& \Delta_{1}=\sum_{i=1}^{5} f_{i}-I_{1}-\xi_{L_{1}}=9-3-6=0 .
\end{aligned}
$$

Because there is only one SOC, the coupling degree of this PM is zero. Moreover, if the revolute joints $\mathrm{R}_{1}^{\prime}$ and $\mathrm{R}_{3}^{\prime}$ of $\mathrm{U}_{1}$ and $\mathrm{U}_{3}$, respectively, and the revolute joints $\mathrm{R}_{2}$ and $\mathrm{R}_{4}^{\prime}$ of $\mathrm{U}_{2}$ and $\mathrm{U}_{4}$, respectively, are made coincident and integrated into revolute joints $R_{13}$ and $R_{24}$, respectively, a new mechanism No.7 in Table 1 can be obtained. For this case, the kinematic chains $\mathrm{U}_{1} \mathrm{P}_{1} \mathrm{~S}_{1}$ and $\mathrm{U}_{3} \mathrm{P}_{3} \mathrm{~S}_{3}$, and $\mathrm{U}_{2} \mathrm{P}_{2} \mathrm{~S}_{2}$ and $\mathrm{U}_{4} \mathrm{P}_{4}$ are converted into $\mathrm{HSOC}_{1}$ and $\mathrm{HSOC}_{2}$, respectively.

$$
\begin{aligned}
& \mathrm{HSOC}_{1}\left\{\mathrm{R}_{13}-\left(\mathrm{R}_{1} \perp \mathrm{P}_{1}-\mathrm{S}_{1}\right)\left(\mathrm{R}_{3} \perp \mathrm{P}_{3}-\mathrm{S}_{3}\right)\right\}, \\
& \mathrm{HSOC}_{2}\left\{\mathrm{R}_{24}-\left(\mathrm{R}_{2} \perp \mathrm{P}_{2}-\mathrm{S}_{2}\right)\left(\mathrm{R}_{4} \perp \mathrm{P}_{4}\right)\right\} .
\end{aligned}
$$

The SOC of the obtained PM can be expressed as

$$
\begin{aligned}
& \mathrm{SOC}_{1}\left\{\mathrm{HSOC}_{1}-\mathrm{HSOC}_{2}\right\}, \\
& \Delta_{1}=\sum_{i=1}^{5} f_{i}-I_{1}-\xi_{L_{1}}=9-3-6=0 .
\end{aligned}
$$

This mechanism also has only one SOC, and its coupling degree is zero. Besides, if the revolute joints $R_{2}^{\prime}$ and $R_{3}^{\prime}$ of $U_{2}$ and $U_{3}$, respectively, in the PM No. $1(b)$ in Table 1 are integrated into one revolute joint $R_{23}$, another new mechanism is obtained, as shown in No. 2 in Table 1.
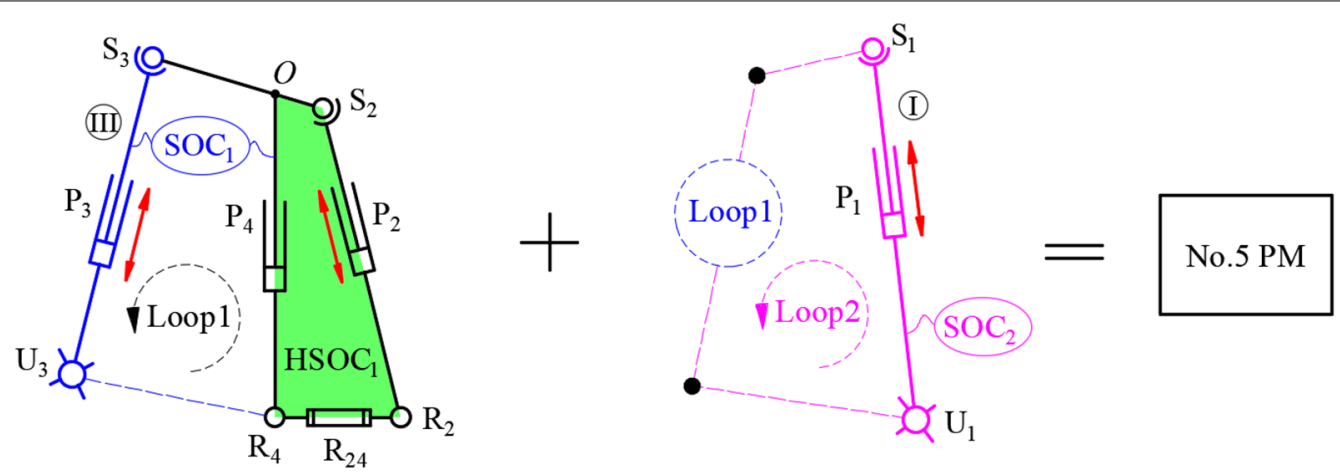

Figure 7 Topological decomposition of No.5 PM 
In this way, the kinematic chains $\mathrm{U}_{2} \mathrm{P}_{2} \mathrm{~S}_{2}$ and $\mathrm{U}_{3} \mathrm{P}_{3} \mathrm{~S}_{3}$ are converted into $\mathrm{HSOC}_{1}$ :

$$
\mathrm{HSOC}_{1}\left\{\mathrm{R}_{23}-\left(\mathrm{R}_{2} \perp \mathrm{P}_{2}-\mathrm{S}_{2}\right)\left(\mathrm{R}_{3} \perp \mathrm{P}_{3}-\mathrm{S}_{3}\right)\right\} .
$$

The SOC and corresponding constraint degree can be expressed as

$$
\begin{aligned}
& \mathrm{SOC}_{1}\left\{\mathrm{HSOC}_{1}-\mathrm{P}_{1}-\mathrm{U}_{1}\right\}, \\
& \Delta_{1}=\sum_{i=1}^{5} f_{i}-I_{1}-\xi_{L_{1}}=9-3-6=0 .
\end{aligned}
$$

It can be proved that the coupling degree of this PM is also zero.

\section{Example}

In this section, the forward kinematic analysis of the obtained R(2RPS\&RP)\&UPS PM is given as an example to verify that a PM with $\kappa=0$ has an explicit form for forward kinematics, which would illustrate the feasibility of the proposed type-synthesis method.

\subsection{Coordinate System}

The schematic diagram of the R(2RPS\&RP)\&UPS PM is shown in Figure 8 , where points $B, B_{1}, B_{2}$, and $B_{3}$ are the centers of joints $\mathrm{R}_{4}, \mathrm{U}_{1}, \mathrm{R}_{2}$, and $\mathrm{R}_{3}$, respectively. Point $O$ is the intersection of the axial axis of the passive limb $R_{4} P_{4}$ and its normal plane in which all centers of $\mathrm{S}$ joints, points $A_{1}, A_{2}$, and $A_{3}$, are placed. A reference frame $B-X Y Z$ is attached to point $B$, satisfying $Y \perp B_{2} B_{3}$ and $Z \perp \triangle B_{1} B_{2} B_{3}$, while a body-fixed frame $O-x y z$ is established at point $O$, satisfying $y \perp A_{2} A_{3}$ and $z \perp \triangle A_{1} A_{2} A_{3}$. The orientation of $O-x y z$ with respect to $B-X Y Z$ can then be evaluated via

$$
\boldsymbol{R}=\operatorname{Rot}\left(X, \theta_{1}\right) \operatorname{Rot}\left(y, \theta_{2}\right)=\left[\begin{array}{ccc}
\mathrm{c} \theta_{2} & 0 & \mathrm{~s} \theta_{2} \\
\mathrm{~s} \theta_{1} \mathrm{~s} \theta_{2} & \mathrm{c} \theta_{1} & -\mathrm{s} \theta_{1} \mathrm{c} \theta_{2} \\
-\mathrm{c} \theta_{1} \mathrm{~s} \theta_{2} & \mathrm{~s} \theta_{1} & \mathrm{c} \theta_{1} \mathrm{c} \theta_{2}
\end{array}\right],
$$

where c and s represent "cos" and "sin", respectively.

\subsection{Forward Kinematics}

When point $O$ is taken as the reference point, its position vector evaluated in $B-X Y Z$ can be expressed as

$$
q_{4} \boldsymbol{s}_{4}=\boldsymbol{b}_{i}+q_{i} \boldsymbol{s}_{i}-\boldsymbol{a}_{i}, \quad i=1,2,3,
$$

where $q_{i}$ is the length, and $\boldsymbol{s}_{i}$ is the unit vector along the axial axis of $\operatorname{limb} I(i=1,2,3,4) ; \boldsymbol{a}_{i}=\boldsymbol{R} \boldsymbol{a}_{i 0} ; \boldsymbol{a}_{i 0}$ and $\boldsymbol{b}_{i}$ are the positioning vectors of $A_{i}$ and $B_{i}(i=1,2,3)$, respectively, measured in $O-x y z$ and $B-X Y Z$, respectively. Noting that

$$
\boldsymbol{s}_{4}=\boldsymbol{R}\left(\begin{array}{lll}
0 & 0 & 1
\end{array}\right)^{\mathrm{T}}=\left(\begin{array}{lll}
\mathrm{s} \theta_{2} & -\mathrm{s} \theta_{1} \mathrm{c} \theta_{2} & \mathrm{c} \theta_{1} \mathrm{c} \theta_{2}
\end{array}\right)^{\mathrm{T}}
$$

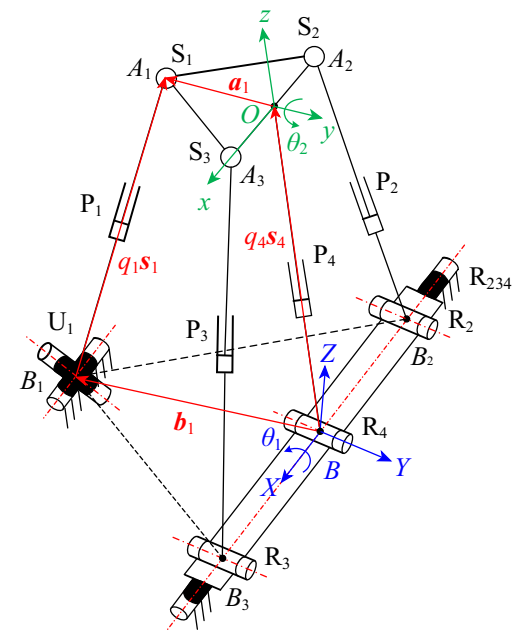

Figure 8 Schematic diagram of R(2RPS\&RP)\&UPS PM

and taking norms on both sides of rearranged Eq. (14) leads to

$$
q_{i}=\left\|q_{4} \boldsymbol{s}_{4}+\boldsymbol{a}_{i}-\boldsymbol{b}_{i}\right\|, \quad i=1,2,3 .
$$

Expanding Eq. (16) then gives the following kinematic equations:

$$
\begin{aligned}
& a_{1}^{2}+b_{1}^{2}+q_{0}^{2}-2 a_{1} b_{1} \mathrm{c} \theta_{1}-2 b_{1} q_{0} \mathrm{~s} \theta_{1} \mathrm{c} \theta_{2}-q_{1}^{2}=0, \\
& a_{2}^{2}+b_{2}^{2}+q_{0}^{2}-2 a_{2} b_{2} \mathrm{c} \theta_{2}-2 b_{2} q_{0} \mathrm{~s} \theta_{2}-q_{2}^{2}=0 \\
& a_{2}^{2}+b_{2}^{2}+q_{0}^{2}-2 a_{2} b_{2} \mathrm{c} \theta_{2}+2 b_{2} q_{0} \mathrm{~s} \theta_{2}-q_{3}^{2}=0
\end{aligned}
$$

where $a_{i}=\left\|\boldsymbol{a}_{i}\right\|$ and $b_{i}=\left\|\boldsymbol{b}_{i}\right\|(i=1,2,3)$, satisfying $a_{2}=a_{3}$ and $b_{2}=b_{3}$. Combining Eq. (18) with Eq. (19) results in

$$
\left\{\begin{array}{l}
\mathrm{c} \theta_{2}=\frac{2\left(a_{2}^{2}+b_{2}^{2}+q_{0}^{2}\right)-q_{2}^{2}-q_{3}^{2}}{4 a_{2} b_{2}} \\
\mathrm{~s} \theta_{2}=\frac{q_{3}^{2}-q_{2}^{2}}{4 b_{2} q_{0}}
\end{array}\right.
$$

Considering that $\mathrm{s}^{2} \theta_{2}+\mathrm{c}^{2} \theta_{2}=1$, we can get

$$
\begin{aligned}
& \left(q_{0}^{2}\right)^{3}+P_{1}\left(q_{0}^{2}\right)^{2}+P_{2} q_{0}^{2}+P_{3}=0, \\
& P_{1}=2 a_{2}^{2}+2 b_{2}^{2}-q_{2}^{2}-q_{3}^{2}, \\
& P_{2}=P_{1}^{2} / 4-4 a_{2}^{2} b_{2}^{2}, \quad P_{3}=a_{2}^{2}\left(q_{3}^{2}-q_{2}^{2}\right)^{2} / 4 .
\end{aligned}
$$

When Eq. (21) is solved, the following equations can be obtained:

$$
q_{0}=\sqrt{\sqrt[3]{C_{1}}+\sqrt[3]{C_{2}}-P_{1} / 3}
$$




$$
\begin{aligned}
& C_{1}=-\frac{D_{2}}{2}+\sqrt{\left(\frac{D_{2}}{2}\right)^{2}+\left(\frac{D_{1}}{3}\right)^{3}} \\
& C_{2}=-\frac{D_{2}}{2}-\sqrt{\left(\frac{D_{2}}{2}\right)^{2}+\left(\frac{D_{1}}{3}\right)^{3}} \\
& D_{1}=P_{2}-P_{1}^{2} / 3, \quad D_{2}=P_{3}+2 P_{1}^{3} / 27-P_{1} P_{2} / 3 .
\end{aligned}
$$

Substituting Eq. (22) into Eq. (20) then leads to

$$
\begin{aligned}
& \theta_{2}=\arcsin \frac{q_{3}^{2}-q_{2}^{2}}{4 b_{2} q_{0}}, \\
& \theta_{1}=2 \arctan \left(\frac{-P_{4}-\sqrt{P_{4}^{2}-P_{6}^{2}+P_{5}^{2}}}{P_{6}-P_{5}}\right), \\
& P_{4}=-2 b_{1} q_{0} \mathrm{c} \theta_{2}, P_{5}=-2 a_{1} b_{1}, P_{6}=a_{1}^{2}+b_{1}^{2}+q_{0}^{2}-q_{1}^{2} .
\end{aligned}
$$

Therefore, the R(2RPS\&RP)\&UPS PM is proven to have an explicit form for forward kinematics.

\section{Conclusions}

Investigation on the type synthesis of $1 \mathrm{~T} 2 \mathrm{R}$ PMs using the method of structure coupling-reducing is presented in this paper. The following conclusions are drawn.

(1) Conducting a topological characteristics analysis of the 3UPS\&UP PM shows that the motions of the three SOCs of the mechanism are highly coupled. Therefore, for this setup, explicit solutions for forward kinematics cannot be achieved.

(2) Aiming at reducing the coupling degree of the 3UPS\&UP PM and synthesizing new mechanisms that have simple forward kinematics, three different methods are proposed, by which eight new PMs having lower coupling degrees are obtained.

(3) The forward kinematic analysis of the R(2RPS\& RP)\&UPS PM is presented. The analysis verifies that the obtained new mechanism, which has a zero coupling degree, uses an explicit form for forward kinematics.

\section{Authors' Contributions}

$\mathrm{HL}$ was in charge of the whole trial; KX wrote the manuscript; XS assisted with the process of analysis; TY and HS provided the assistance of theory. All authors read and approved the final manuscript.

\section{Authors' Information}

Haitao Liu, born in 1981, is currently a professor at Tianjin University, China. He received his PhD degree from Tianjin University, China, in 2010. His research interests include hybrid robot and intelligent robotics.

Ke Xu, born in 1992, is currently a PhD candidate at Key Laboratory of Mechanism Theory and Equipment Design, Ministry of Education, Tianjin University, China. He received his master degree on mechanical design and theory from Changzhou University, China, in 2018.

Huiping Shen, born in 1965, is currently a professor at School of Mechanical Engineering, Changzhou University, China. His research interests include parallel mechanism and mechatronics.

Xianlei Shan, born in 1987, is currently a postdoctor at Key Laboratory of Mechanism Theory and Equipment Design, Ministry of Education, Tianjin University, China.

Tingli Yang, born in 1940, is currently a distinguished professor at Changzhou University, China.

\section{Competing Interests}

The authors declare that they have no competing interests.

\section{Funding}

Supported by National Key R\&D program of China (Grant No. 2017YFB1301800), National Natural Science Foundation of China (Grant No. 51622508), and National Defense Basic Scientific Research program of China (Grant No. JCKY2017203B066)

\section{Author Details}

${ }^{1}$ Key Laboratory of Mechanism Theory and Equipment Design, Ministry of Education, Tianjin University, Tianjin 300350, China. ${ }^{2}$ School of Mechanical Engineering, Changzhou University, Changzhou 213164, China.

Received: 17 January 2019 Revised: 5 September 2019 Accepted: 18 October 2019

Published online: 06 November 2019

\section{References}

[1] Z Huang, Y S Zhao, T S Zhao. Advanced spatial mechanism. Higher Education Press, 2014

[2] X L Yang, HTWu, Y Li, et al. A dual quaternion solution to the forward kinematics of a class of six-DOF parallel robots with full or reductant actuation. Mechanism and Machine Theory, 2017, 107: 27-36.

[3] W Zhou, W Chen, H Liu, et al. A new forward kinematic algorithm for a general Stewart platform. Mechanism and Machine Theory, 2015, 87: 177-190.

[4] J S Kim, Y H Jeong, J H Park. A geometric approach for forward kinematics analysis of a 3-SPS/S redundant motion manipulator with an extra sensor using conformal geometric algebra. Meccanica, 2016, 51(10): 2289-2304.

[5] X Wu, Z Xie. Forward kinematics analysis of a novel 3-DOF parallel manipulator. Scientia Iranica, 2019, 26(1): 346-357.

[6] R R Serrezuela, M Á T Cardozo, D L Ardila, et al. A consistent methodology for the development of inverse and direct kinematics of robust industrial robots. ARPN Journal of Engineering and Applied Sciences, 2018, 13(01): 293-301.

[7] W Li, J Angeles. A novel three-loop parallel robot with full mobility: kinematics, singularity, workspace, and dexterity analysis. Journal of Mechanisms and Robotics, 2017, 9(5): 051003.

[8] J P Merlet. A generic numerical continuation scheme for solving the direct kinematics of cable-driven parallel robot with deformable cables. 2016 IEEE/RSJ International Conference on Intelligent Robots and Systems (IROS), 2016: 4337-4343.

[9] A Zubizarreta, M Larrea, E Irigoyen, et al. Real time direct kinematic problem computation of the 3PRS robot using neural networks. Neurocomputing, 2018, 271: 104-114. 
[10] S Yang, T Sun, T Huang. Type synthesis of parallel mechanisms having 3T1R motion with variable rotational axis. Mechanism and Machine Theory, 2017, 109: 220-230.

[11] Y Xu, D Zhang, J Yao, et al. Type synthesis of the 2R1T parallel mechanism with two continuous rotational axes and study on the principle of its motion decoupling. Mechanism and Machine Theory, 2017, 108: 27-40

[12] Y Song, P Han, P Wang. Type synthesis of 1T2R and 2R1T parallel mechanisms employing conformal geometric algebra. Mechanism and Machine Theory, 2018, 121: 475-486.

[13] T L Yang, A X Liu, Y F Luo, et al. Theory and application of robot mechanism topology. Beijing: Science Press, 2012. (in Chinese)

[14] T L Yang, A X Liu, H P Shen, et al. Topology design of robot mechanisms. Springer, Singapore, 2018.

[15] H P Shen, L J Yang, X R Zhu, et al. A Method for structure couplingreducing of parallel mechanisms. The 2015 International Conference on Intelligent Robotics and Applications, Portsmouth, UK, August 24-27, 2015.

[16] H P Shen, X R Zhu, H B Yin, et al. Principle and design method for structure coupling-reducing of parallel mechanisms. Journal of Mechanical Engineering, 2016, 47(8): 388-398. (in Chinese)

[17] H P Shen, L Z Ma, T L Yang. Kinematic structural synthesis of 3-translational weakly-coupled parallel mechanisms based on hybrid chains. Mechanism and Machine Theory, 2005, 41(4): 22-27.

[18] H P Shen, H C Qiang, Q F Zeng, et al. Topological design for a class of novel 3T1R parallel mechanisms with low coupling degree based on coupling-reducing. China Mechanical Engineering, 2017, 28(10): 11631171. (in Chinese)

[19] H P Shen, T L Yang, L Z Ma. Methodology for type synthesis of kinematic structures of 6-DOF weakly-coupling parallel mechanisms. Journal of Mechanical Engineering, 2004, 40(7): 14-19. (in Chinese)

[20] H P Shen, T L Yang, L Z Ma. Synthesis and structure analysis of kinematic structures of 6-dof parallel robotic mechanisms. Mechanism and Machine Theory, 2005, 40(10): 1164-1180.

[21] T ZYu, H P Shen, J M Deng, et al. An easily manufactured structure and its analytic solutions for forward and inverse position of 1-2-3-SPS type
6-DOF basic parallel mechanism. The 2012 IEEE International Conference on Robotics and Biomimetic, December, 11-14, Guangzhou, 2012.

[22] Z Wang, H P Shen, J M Deng, et al. An easily manufactured 6-DOF 3-1-1-1 SPS type parallel mechanism and its forward kinematics. The 2nd IFTOMM Symposium on Mechanism Design for Robotics, October, 12-14, Beijing, 2012.

[23] H P Shen, H B Yin, Z Wang, et al. Research on forward position solutions for 6-SPS parallel mechanisms based on topology structure analysis. Journal of Mechanical Engineering, 2013, 49(21): 70-80. (in Chinese)

[24] S A Joshi, LW Tsai. The kinematics of a class of 3-DOF, 4-legged parallel manipulators. ASME Journal of Mechanical Design, 2003, 125: 52-60.

[25] B Siciliano. The Tricept robot: inverse kinematics, manipulability analysis and closed-loop direct kinematics algorithm. Robotica, 1999, 17(4): 437-455.

[26] S Fan, S Fan, X Zhang. A hybrid optimization method of dimensions for the Tricept parallel robot. International Conference on Mechanical Design, 2017: 1343-1364

[27] T L Yang, A X Liu, Y F Luo, et al. Position and orientation characteristic equation for topological design of robot mechanisms. Journal of Mechanical Design, 2009, 131(2): 021001.

[28] T L Yang, A X Liu, H P Shen, et al. On the correctness and strictness of the $P O C$ equation for topological structure design of robot mechanisms. Journal of Mechanisms and Robotics, 2013, 5(2): 021009.

[29] T L Yang, A X Liu, H P Shen, et al. Composition principle based on SingleOpen-Chain unit for general spatial mechanisms and its application. Journal of Mechanisms and Robotics, 2018, 10(5): 051005.

[30] THuang, M Li, X M Zhao, et al. Conceptual design and dimensional synthesis for a 3-DOF module of TriVariant-a novel 5-DOF reconfigurable hybrid robot. IEEE Transactions on Robotics, 2005, 21(3): 449-456.

[31] C L Dong, H T Liu, W Yue, et al. Stiffness modeling and analysis of a novel 5-DOF hybrid robot. Mechanism and Machine Theory, 2018, 125: 80-93.

\section{Submit your manuscript to a SpringerOpen ${ }^{\odot}$ journal and benefit from:}

- Convenient online submission

- Rigorous peer review

- Open access: articles freely available online

- High visibility within the field

- Retaining the copyright to your article

Submit your next manuscript at $\boldsymbol{\nabla}$ springeropen.com 